\title{
Ser1333 phosphorylation indicates ROCKI activation
}

\author{
Hsiang-Hao Chuang ${ }^{1}$, Shao-Wei Liang ${ }^{2}$, Zee-Fen Chang ${ }^{1 *}$ and Hsiao-Hui Lee ${ }^{2^{*}}$
}

\begin{abstract}
Background: Two isoforms of Rho-associated protein kinase (ROCK), ROCKI and ROCKII, play a pivotal role in regulation of cytoskeleton and are involved in multiple cellular processes in mammalian cells. Knockout mice experiments have indicated that the functions of ROCKI and II are probably non-redundant in physiology. However, it is difficult to differentiate the activation status of ROCKI and ROCKII in biological samples. Previously, we have identified phosphorylation site of ROCKII at Ser1366 residue sensitive to ROCK inhibition. We further investigated the activity-dependent phosphorylation site in ROCKI to establish the reagents that can be used to detect their individual activation.

Results: The phosphorylation site of ROCKI sensitive to its inhibition was identified to be the Ser1333 residue. The ROCKI pSer1333-specific antibody does not cross-react with phosphorylated ROCKII. The extent of S1333 phosphorylation of ROCKI correlates with myosin II light chain phosphorylation in cells in response to RhoA stimulation.
\end{abstract}

Conclusions: Active ROCKI is phosphorylated at Ser1333 site. Antibodies that recognize phospho-Ser1333 of ROCKI and phospho-S1366 residues of ROCKII offer a means to discriminate their individual active status in cells and tissues.

Keywords: Rho-associated protein kinase (ROCK), RhoA, Marker

\section{Background}

Two isoforms of Rho-associated protein kinase (ROCK), ROCKI (also called ROK $\beta$ ) and ROCKII (also known as Rho kinase and ROK $\alpha$ ) have been identified as RhoA-GTP interacting proteins in mammals [1,2]. They are serine/ threonine kinases important for regulation of actin dynamics and cytoskeleton organization [3-5]. These two human kinases share $64 \%$ homology in amino acid sequence with $89 \%$ identity in the catalytic kinase domain [5]. They contain a Rho-binding domain (RBD) in the coiled-coil region and a pleckstrin homology $(\mathrm{PH})$ domain in the C-terminal region, which folds back onto the N-terminal kinase domain to autoinhibit kinase functions. GTP-RhoA binding relieves the auto-inhibition, switching-on the kinase activity [6]. ROCKI and ROCKII have common substrates, such as myosin light chain

\footnotetext{
* Correspondence: zfchang@ym.edu.tw; hhl@ym.edu.tw

'Institute of Biochemistry and Molecular Biology, National Yang-Ming University, No. 155, Sec. 2, Linong St, Taipei 11221, Taiwan

${ }^{2}$ Department of Life Sciences and Institute of Genome Sciences, National Yang-Ming University, No. 155, Sec. 2, Linong St, Taipei 11221, Taiwan
}

(MLC), myosin binding subunit (MYPT1) of the MLC phosphatase, LIM kinases (LIMK1 and LIMK2), $\alpha-$ adducin, ezrin-radixin-moesin (ERM) proteins, and etc. [4,5,7-9]. Collectively, the kinase activation promotes the stabilization of actin filaments and myosin activity to increase actomyosin-driven cellular contractility $[10,11]$. In addition to regulation by RhoA binding, ROCKs are negatively regulated by distinct binding proteins or phosphorylation. For example, Gem and RhoE specifically inhibit ROCKI and Rad for ROCKII inhibition [5,12]. ROCKII has been shown to be phosphorylated at Tyr722 residue by Src kinase to decrease its affinity to GTP-RhoA [13], and dephosphorylated by Shp2 phosphatase [14]. Therefore, the activity of ROCKI and II in vivo could be highly dependent on the cellular context.

To know the distinct biological roles of ROCKI and ROCKII, the $\mathrm{ROCKI}^{-1-}$ and $\mathrm{ROCKII}^{-1-}$ mice have been generated $[15,16]$. $\mathrm{ROCKI}^{-/-}$mice are postnatal lethal, because of impairment of umbilical ring closure [16], and $\mathrm{ROCKII}^{-/-}$mice are embryonic lethal at the percentage of $90 \%$ due to the dysfunction of placenta and

\section{Ciomed Central}


intrauterine growth retardation caused by thrombus formation in the labyrinth layer of placenta [15]. These studies suggest that ROCKI and ROCKII have distinct functions in development.

Many reports have highlighted the association of ROCK activation with cancer progression and suggest the potential of ROCK as therapeutic targets in cancer [17-19]. The level of ROCKI RNA in tumor tissue correlates with the tumor grade and poor overall survival in breast cancer patients [20], and higher level of ROCKI protein has been found in osteosarcoma tissues [21]. As for ROCKII, higher expression has been reported in aggressive hepatocellular carcinomas, colon and bladder cancers [22-24]. Considering that the expression level at mRNA or protein of ROCK may not be necessarily correlated with their kinase activity, we developed the reagents that can directly and specifically detect the activation status of ROCKI and ROCKII in cells and tissues by identification of their corresponding phosphorylation sites. Our previous results have provided evidence that ROCKII at Ser1366 residue reflects its kinase activation [25]. In this study, we further showed activated ROCKI with phosphorylation at Ser1333 residue. Thus, the specific antibodies, one against ROCKI Ser1333 phosphorylation and another against ROCKII Ser1366 phosphorylation, can be used to detect the active form of ROCKI and ROCKII, respectively.

\section{Methods}

\section{Plasmids and reagents}

The S1333A mutation of ROCKI was introduced to wildtype pCMV2-flag-ROCKI described previously [25] using the Quick-Change site-directed mutagenesis kit (Stratagene). Y27632 was from Calbiochem-Novabiochem Corp.; $\lambda$ PPase was from New England Biolabs; nocodazole, antiflag and anti-MLC antibodies were from Sigma-Aldrich; anti-ROCKI, anti-ROCKII and anti-RhoA antibodies were purchased from Santa Cruz Biotechnology; antiphospho-MLC2 (T18/S19) antibody from Cell Signaling Technology; anti-pSer1366 ROCKII antibody was described previously [25].

\section{Cell culture and transient transfection}

Normal mouse embryonic fibroblasts (MEFs) and HEK293T cells were maintained in Dulbecco's modified Eagle's medium (DMEM) supplemented with $10 \%(\mathrm{v} / \mathrm{v})$ fetal bovine serum (FBS) in a humidified atmosphere of $5 \% \mathrm{CO}_{2} / 95 \%$ air at $37^{\circ} \mathrm{C}$. For transient transfection experiments, HEK293T cells were transfected by PolyJet reagent (SignaGen Laboratories).

\section{Immunoprecipitation and in vitro kinase reaction}

Flag-ROCKI-expressing cells were harvested in an IP buffer (1\% NP-40, 5\% glycerol, $50 \mathrm{mM}$ Tris-HCl, pH 7.4, $150 \mathrm{mM} \mathrm{NaCl}, 1 \mathrm{mM}$ PMSF, $50 \mathrm{mM} \mathrm{NaF}, 2 \mathrm{mM}$
$\mathrm{Na}_{3} \mathrm{VO}_{4}$ and protease inhibitor cocktail). The lysates after pre-clearance were incubated with anti-flag antibody conjugated agarose beads (Sigma-Aldrich) at $4^{\circ} \mathrm{C}$ for $1 \mathrm{hr}$. The immunoprecipitates were pre-incubated with or without $100 \mu \mathrm{M}$ of Y27632, which was followed by incubation with a kinase buffer $(50 \mathrm{mM}$ Tris- $\mathrm{HCl}, \mathrm{pH} 7.4,10 \mathrm{mM}$ $\mathrm{MgCl}_{2}, 1 \mathrm{mM}$ EGTA, $0.5 \mathrm{mM}$ DTT, $5 \mathrm{mM} \mathrm{NaF}, 0.1 \mathrm{mM}$ $\mathrm{Na}_{3} \mathrm{VO}_{4}$, and $20 \mu \mathrm{M}$ ATP) containing $5 \mu \mathrm{Ci}$ of $\left[\gamma_{-}{ }^{32} \mathrm{p}\right] \mathrm{ATP}$ at $30^{\circ} \mathrm{C}$ for $20 \mathrm{~min}$. The reaction was stopped and products were separated by SDS-PAGE, transferred to a PVDF membrane. The phosphorylation status and amounts of the proteins were detected by autoradiography and Western blotting with anti-ROCKI antibody, respectively.

\section{Phospho-specific antibody generation}

The polyclonal anti-pS1333 ROCKI antibody was raised using phosphopeptide containing phosphorylated Ser1333 of ROCKI conjugated with keyhole limpet haemocyanin (KLH) as an antigen to immunize rabbits. Anti-sera were collected and sequentially affinity purified by phosphopeptide- and non-phosphopeptideconjugated columns (ICON Biotechnology Co., Itd., Taiwan).

\section{Results and discussion}

Identification of activity-dependent phosphorylation site of ROCKI

To search for kinase-dependent phosphorylation site of ROCKI, we performed an in vitro kinase reaction using immunoprecipitated flag-tagged ROCKI protein in the presence of $\left[\gamma^{3}{ }^{32} \mathrm{P}\right]$ ATP. Autoradiography detected the phosphorylation signal, which was abolished by including ROCK inhibitor Y27632 in the in vitro kinase reaction (Figure 1A). Ser1333 residue in human ROCKI sequence is corresponding to the Ser1366 of ROCKII and is conserved in vertebrates. We then isolated immunocomplex of wild-type (WT) and S1333A mutant of flag-ROCKI proteins in cells for the in vitro kinase assay using $\left[\gamma^{-32} \mathrm{P}\right]$ ATP labeling. The result showed that S1333A mutation markedly reduced the intensity of $\left[\gamma_{-}{ }^{32} \mathrm{P}\right] \mathrm{ATP}$ labeling in ROCKI (Figure 1B), indicating that Ser1333 residue is one of the phosphorylation sites of ROCKI dependent on its own kinase activity.

\section{Validation of Ser1333 phosphorylation of ROCKI by specific antibody}

To assure that phosphorylation at Ser1333 is a mark of ROCKI activation in biological samples, we then generated a phospho-specific antibody by a phosphopeptide containing pSer1333 of ROCKI. The specificity of this antibody was tested by Western blot analysis of the immunoprecipitated WT and S1333A mutant of flagROCKI proteins. The results showed that purified antipS1333 ROCKI antibody was capable of detecting the 

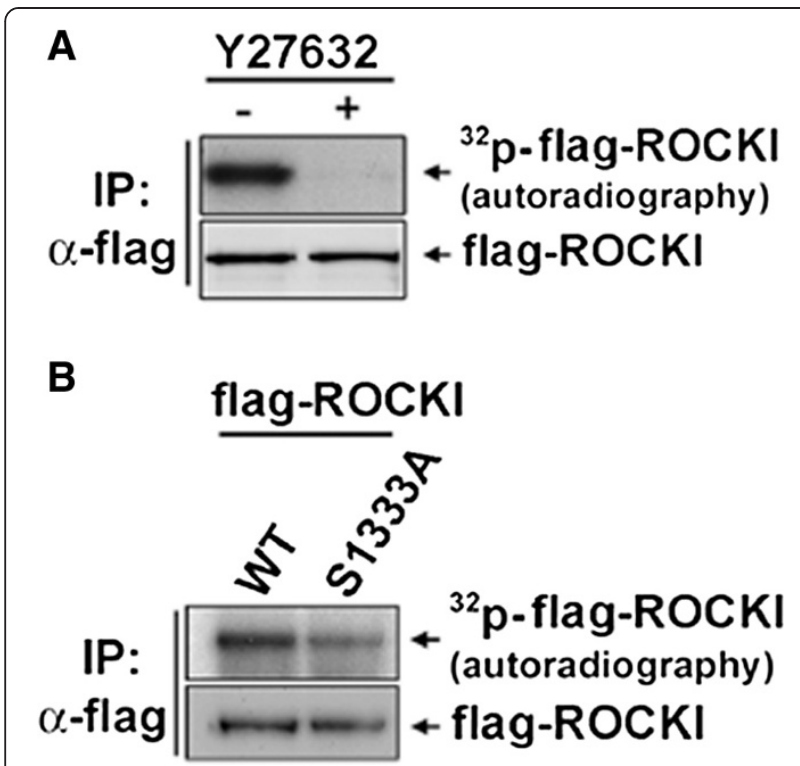

Figure 1 ROCKI is phosphorylated at Ser1333 residue. (A) The flag-ROCKI protein was expressed in HEK293T cells and immunoprecipitated with anti-flag antibody. Equal amounts of immunoprecipitated complex were pre-incubated with $100 \mu \mathrm{M}$ of Y27632 for $20 \mathrm{~min}$ and subjected to an in vitro kinase reaction with

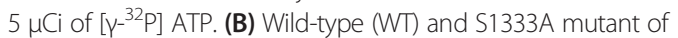
flag-ROCKI proteins were immunoprecipitated for in vitro kinase reaction. After 20 min, the reaction was stopped and proteins were then applied to SDS-PAGE followed by autoradiography. The protein level of flag-ROCKI was determined by Western blotting with anti-ROCKl antibody.

phosphorylation of immunoprecipitated WT but not S1333A flag-ROCKI protein. The signal was neutralized by phosphorylated peptide but not by non-phosphorylated peptide (Figure 2A). Treatment of flag-ROCKI (WT) immunoprecipitates with $\lambda$ protein phosphatase ( $\lambda$ PPase) abolished the signal (Figure 2B). These data indicate the specificity of anti-pSer1333 ROCKI antibody. Given the similarity in amino acid sequence surrounding Ser1333 in ROCKI and Ser1366 in ROCKII, we used flag-tagged ROCKI and ROCKII immunoprecipitates to verify the specificity of these two antibodies. As shown in Figure 2C, neither did anti-pSer1333 ROCKI antibody cross-react with ROCKII, nor anti-pSer1366 ROCKII antibody to ROCKI.

\section{Detection of endogenous ROCKI activation by assessing Ser1333 phosphorylation}

We further used this antibody for direct Western blot analysis of lysates from HEK293T cells expressing WT and S1333A ROCKI. As shown in Figure 3A, the antibody detected a major signal in WT ROCKI but not S1333A mutant. A lower band was a non-specific signal because the intensity was similar regardless of the ectopic expression of ROCKI. Next, we assessed the change in Ser1333 phosphorylation of endogenous ROCKI in response to RhoA activation. To this end, HEK293T cells were transfected with the expression construct of GFP-RhoAV14, a constitutively active form, GFP-RhoAN19, a dominant negative form, or GFP-RhoAV14E40L, a constitutive active mutant defective in interaction with ROCK [26], for Western blot analysis. The level of Ser1333 phosphorylation of ROCKI in cells was increased by expression of GFP-RhoAV14. However, expression of GFP-RhoAV14E40L had no effect on the level Ser1333 phosphorylation of ROCKI. Expression of GFP-RhoAN19 reduced ROCKI Ser1333 phosphorylation (Figure 3B). These data suggest that the activation of endogenous ROCKI by RhoA can be specifically detected by Western blot analysis using anti-pSer1333 ROCKI antibody.
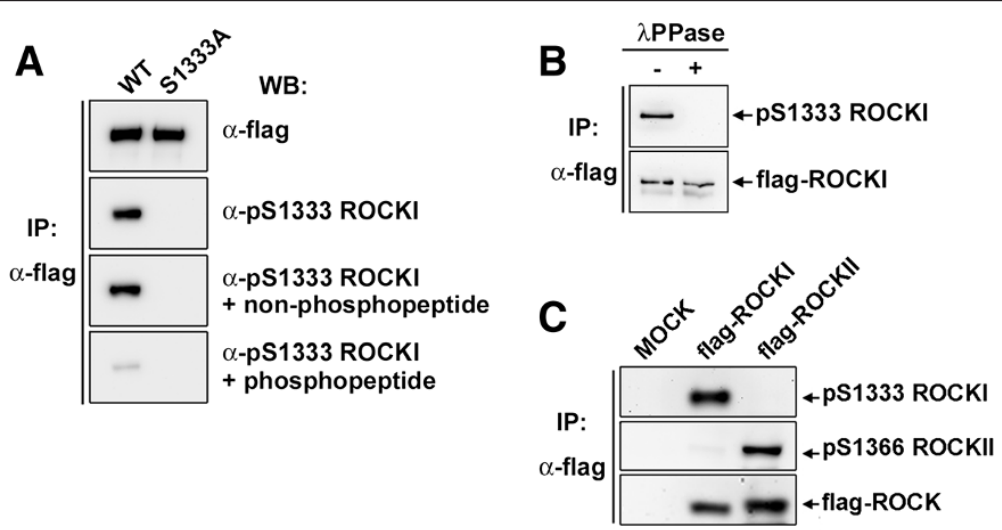

Figure 2 Validation of anti-pSer1333 ROCKI antibody. (A) Wild-type (WT) or S1333A mutant of flag-ROCKI proteins were expressed in HEK293T cells and immunoprecipitated with anti-flag antibody followed by probed with anti-flag or anti-pSer1333 ROCKI antibody with or without peptide competition in a same set of sample. (B) WT flag-ROCKI immunoprecipitates were incubated with or without $\lambda$ PPase at $30^{\circ} \mathrm{C}$ for 20 min followed by Western blot analysis as indicated. (C) Flag-tagged ROCKI and ROCKII proteins were immunoprecipitated for Western blot analysis using anti-pSer1333 ROCKI, anti-pSer1366 ROCKII, and anti-flag antibodies in a same set of sample. 


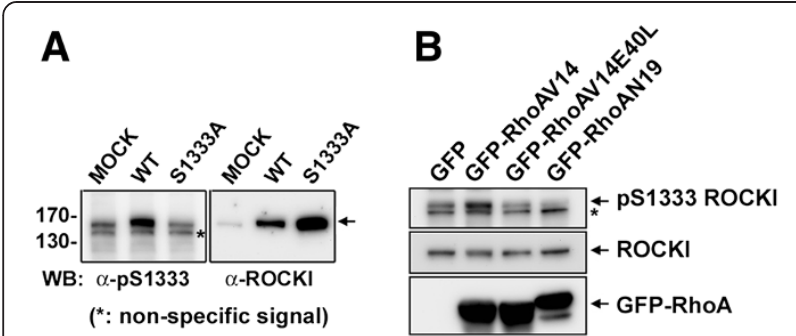

Figure 3 Detection of endogenous ROCKI phosphorylation at Ser1333 residue. (A) HEK293T cells expressing WT or S1333A mutant of flag-ROCKI were harvested for Western blot analysis with anti-pS1333 ROCKI and anti-ROCKI antibodies. (B) Cells were transfected with the expression constructs of GFP, GFP-RhoAV14, GFP-RhoAV14E40L, or GFP-RhoAN19 and harvested for Western blotting with antibodies as indicated. ${ }^{*}$, non-specific signal.

We also examined the changes of ROCKI S1333 phosphorylation in serum-starved mouse embryonic fibroblasts (MEFs) that were treated with nocodazole to activate the RhoA signal via GEF-H1 released from microtubules disassembly [27]. As shown in Figure 4, ROCKI Ser1333 phosphorylation level was dramatically increased (5-fold) by nocodazole treatment at $15 \mathrm{~min}$ as seen in the increase of MLC phosphorylation. Similarly, the phosphorylation of ROCKII at Ser1366 was also increased. Treatment of cells with Y27632 prevented both nocodazole-induced ROCKI S1333 and ROCKII S1366 phosphorylation, suggesting the increase of the phosphorylation signal correlates with their corresponding kinase activation. The Y27632 treatment did not completely abolish the basal levels of ROCKI S1333 phosphorylation and ROCKII S1366 phosphorylation. It

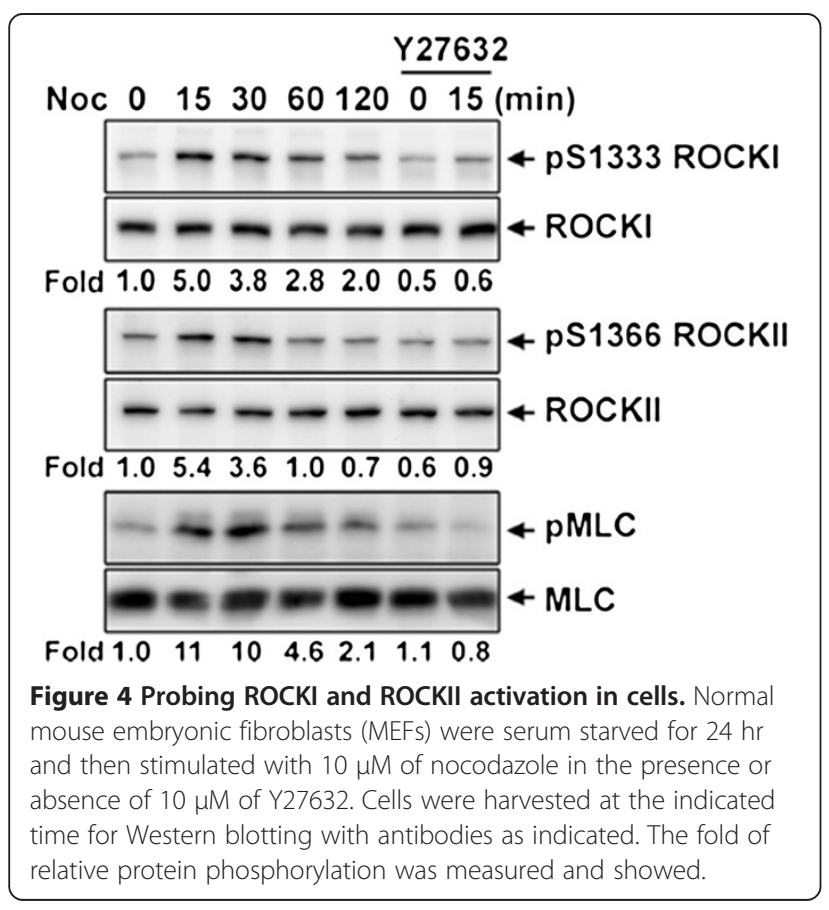

is uncertain whether this is due to the incomplete inhibition of ROCK kinase or a background level of nonspecific signal. Nevertheless, the stimulation effect is reflected by these phosphorylation marks. Interestingly, the level of ROCKII S1366 phosphorylation was declined to the basal level at $60 \mathrm{~min}$, while ROCKI S1333 phosphorylation sustained to $120 \mathrm{~min}$. Thus, probing ROCKI Ser1333 and ROCKII Ser1366 phosphorylation is able to discriminate the differences in the kinetic of ROCKI and ROCKII activation in cells.

It is known that ROCKs form dimer [28-32]. In this study, we did not examine whether ROCKI S1333 phosphorylation is due to ROCKII in the heterodimer. It has been reported that the dimer consisting of wild-type kinase and catalytic-dead ROCK kinase domain is catalytically inactive [30]. Therefore, we are unable to test whether ROCKII can phosphorylate catalytic-dead ROCKI at S1333 in the heterodimer form. In an overexpression experiment, we found that the amount of homodimer of ROCKII was more than 10-fold higher than that of ROCKI/II (data not shown). Considering that all ROCKs purified from a variety of tissues have been shown to be in homodimer form [2,33-35], the physiological significance of S1333 phosphorylation in the heterodimer form of ROCKI/II is probably negligible. We also cannot exclude the possibility that other kinase is able to phosphorylate the S1333 site. Nevertheless, the correlation of S1333 phosphorylation with its upstream RhoA regulation and the extent of downstream substrate MLC phosphorylation suggest this modification as an indicator of ROCKI activation.

Small-molecule inhibitors against ROCK, such as Y27632 and Fasudil, have been developed to have potential in clinical implication $[17,36,37]$. Increasing number of clinical trials and animal experiments using these inhibitors suggest that ROCK activation plays an important role in the pathogenesis of many cardiovascular diseases, neurological disorders and cancers [3,4,38-43]. Although the functions of ROCKI and ROCKII are analogous and compensatory, genetic deletion studies suggest that each kinase might play distinct roles depending on tissue types and certain biological processes. Also unknown is which ROCK isoform is responsible for pathogenesis of a specific tissue in diseases and related to disease progression. Our antibodies that can detect the phosphorylation of ROCKI at Ser1333 and ROCKII at Ser1366 offer new opportunities to differentiate the activation status of ROCKI and II in association with diseases. Of note, all the current inhibitors cannot discern between ROCKI and II. The antibodies that can detect active forms of ROCKI and II provide valuable tools for screening ROCKI and ROCKII specific inhibitors.

\section{Conclusion}

Ser1333 phosphorylation can indicate the active status of ROCKI in response to RhoA signaling. Thus, antibodies 
that recognize phosphorylation at Ser1333 and S1366 residues of ROCKI and II, respectively, are capable of probing their corresponding activation in biological samples. Also, these antibodies might be very useful reagents for drug screening of inhibitors specific against ROCKI and ROCKII isoform.

\section{Competing interests}

The authors declare that they have no competing interests.

\section{Authors' contributions}

HHC and SWL performed experiments. HHL and ZFC designed the study and wrote the paper. All authors read and approved the final paper.

\section{Acknowledgments}

This research is supported by Taiwan National Science Council [grant number NSC102-2628-B-010-003-MY3], and in part by the UST-UCSD International Center of Excellence in Advanced Bio-engineering sponsored by the Taiwan National Science Council I-RiCE Program [grant number NSC102-2911-I-009-101]. We also are grateful for the support from the Ministry of Education in National Yang-Ming University, Aim for the Top University Plan.

Received: 2 July 2013 Accepted: 22 October 2013

Published: 29 October 2013

\section{References}

1. Fujisawa K, Fujita A, Ishizaki T, Saito Y, Narumiya S: Identification of the Rho-binding domain of p160ROCK, a Rho-associated coiled-coil containing protein kinase. J Biol Chem 1996, 271:23022-23028.

2. Matsui T, Amano M, Yamamoto T, Chihara K, Nakafuku M, Ito M, Nakano T, Okawa K, Iwamatsu A, Kaibuchi K: Rho-associated kinase, a novel serine/threonine kinase, as a putative target for small GTP binding protein Rho. EMBO J 1996, 15:2208-2216.

3. Mueller BK, Mack $\mathrm{H}$, Teusch N: Rho kinase, a promising drug target for neurological disorders. Nat Rev Drug Discov 2005, 4:387-398.

4. Rikitake Y, Liao JK: ROCKs as therapeutic targets in cardiovascular diseases. Expert Rev Cardiovasc Ther 2005, 3:441-451.

5. Riento K, Ridley AJ: Rocks: multifunctional kinases in cell behaviour. Nat Rev Mol Cell Biol 2003, 4:446-456.

6. Amano M, Chihara K, Nakamura N, Kaneko T, Matsuura Y, Kaibuchi K: The $\mathrm{COOH}$ terminus of Rho-kinase negatively regulates rho-kinase activity. J Biol Chem 1999, 274:32418-32424

7. Amano M, Ito M, Kimura K, Fukata Y, Chihara K, Nakano T, Matsuura Y, Kaibuchi K: Phosphorylation and activation of myosin by Rho-associated kinase (Rho-kinase). J Biol Chem 1996, 271:20246-20249.

8. Kimura K, Ito M, Amano M, Chihara K, Fukata Y, Nakafuku M, Yamamori B, Feng J, Nakano T, Okawa K, et al: Regulation of myosin phosphatase by Rho and Rho-associated kinase (Rho-kinase). Science 1996, 273:245-248.

9. Amano M, Fukata Y, Kaibuchi K: Regulation and functions of Rho-associated kinase. Exp Cell Res 2000, 261:44-51.

10. Ridley AJ, Hall A: The small GTP-binding protein rho regulates the assembly of focal adhesions and actin stress fibers in response to growth factors. Cell 1992, 70:389-399.

11. Chrzanowska-Wodnicka M, Burridge K: Rho-stimulated contractility drives the formation of stress fibers and focal adhesions. J Cell Biol 1996, 133:1403-1415.

12. Ward Y, Yap SF, Ravichandran V, Matsumura F, Ito M, Spinelli B, Kelly K: The GTP binding proteins Gem and Rad are negative regulators of the Rho-Rho kinase pathway. J Cell Biol 2002, 157:291-302.

13. Lee HH, Tien SC, Jou TS, Chang YC, Jhong JG, Chang ZF: Src-dependent phosphorylation of ROCK participates in regulation of focal adhesion dynamics. J Cell Sci 2010, 123:3368-3377.

14. Lee HH, Chang ZF: Regulation of RhoA-dependent ROCKII activation by Shp2. J Cell Biol 2008, 181:999-1012.

15. Thumkeo D, Keel J, Ishizaki T, Hirose M, Nonomura K Oshima H, Oshima M, Taketo MM, Narumiya S: Targeted disruption of the mouse rho-associated kinase 2 gene results in intrauterine growth retardation and fetal death. Mol Cell Biol 2003, 23:5043-5055.

16. Shimizu Y, Thumkeo D, Keel J, Ishizaki T, Oshima H, Oshima M, Noda Y, Matsumura F, Taketo MM, Narumiya S: ROCK-I regulates closure of the eyelids and ventral body wall by inducing assembly of actomyosin bundles. J Cell Biol 2005, 168:941-953.

17. Olson MF: Applications for ROCK kinase inhibition. Curr Opin Cell Biol 2008, 20:242-248.

18. Rath N, Olson MF: Rho-associated kinases in tumorigenesis: re-considering ROCK inhibition for cancer therapy. EMBO Reports 2012, 13:900-908

19. Morgan-Fisher M, Wewer UM, Yoneda A: Regulation of ROCK activity in cancer. J Histochem Cytochem 2013, 61:185-198.

20. Lane J, Martin TA, Watkins G, Mansel RE, Jiang WG: The expression and prognostic value of ROCK I and ROCK II and their role in human breast cancer. Int J Oncol 2008, 33:585-593.

21. Liu X, Choy E, Hornicek FJ, Yang S, Yang C, Harmon D, Mankin H, Duan Z: ROCK1 as a potential therapeutic target in osteosarcoma. J Orthop Res 2011, 29:1259-1266.

22. Vishnubhotla R, Sun S, Huq J, Bulic M, Ramesh A, Guzman G, Cho M, Glover SC: ROCK-II mediates colon cancer invasion via regulation of MMP-2 and MMP-13 at the site of invadopodia as revealed by multiphoton imaging. Lab Invest 2007, 87:1149-1158.

23. Kamai T, Tsujii T, Arai K, Takagi K, Asami H, Ito Y, Oshima H: Significant association of Rho/ROCK pathway with invasion and metastasis of bladder cancer. Clin Cancer Res 2003, 9:2632-2641.

24. Wong CC, Wong CM, Tung EK, Man K, Ng IO: Rho-kinase 2 is frequently overexpressed in hepatocellular carcinoma and involved in tumor invasion. Hepatology 2009, 49:1583-1594.

25. Chuang HH, Yang CH, Tsay YG, Hsu CY, Tseng LM, Chang ZF, Lee HH: ROCKII Ser1366 phosphorylation reflects the activation status. Biochem J 2012, 443:145-151.

26. Sahai E, Alberts AS, Treisman R: RhoA effector mutants reveal distinct effector pathways for cytoskeletal reorganization, SRF activation and transformation. EMBO J 1998, 17:1350-1361.

27. Krendel M, Zenke FT, Bokoch GM: Nucleotide exchange factor GEF-H1 mediates cross-talk between microtubules and the actin cytoskeleton. Nat Cell Biol 2002, 4:294-301.

28. Jacobs M, Hayakawa K, Swenson L, Bellon S, Fleming M, Taslimi P, Doran J: The structure of dimeric ROCKI reveals the mechanism for ligand selectivity. J Biol Chem 2006, 281:260-268.

29. Dvorsky $R$, Blumenstein $L$, Vetter IR, Ahmadian MR: Structural insights into the interaction of ROCKI with the switch regions of RhoA. J Biol Chem 2004, 279:7098-7104

30. Chen XQ, Tan I, Ng CH, Hall C, Lim L, Leung T: Characterization of RhoAbinding kinase ROKalpha implication of the pleckstrin homology domain in ROKalpha function using region-specific antibodies. J Biol Chem 2002, 277:12680-12688.

31. Doran JD, Liu X, Taslimi P, Saadat A, Fox T: New insights into the structurefunction relationships of Rho-associated kinase: a thermodynamic and hydrodynamic study of the dimer-to-monomer transition and its kinetic implications. Biochem J 2004, 384:255-262.

32. Yamaguchi H, Kasa M, Amano M, Kaibuchi K, Hakoshima T: Molecular mechanism for the regulation of rho-kinase by dimerization and its inhibition by fasudil. Structure 2006, 14:589-600

33. Amano M, Fukata $Y$, Shimokawa H, Kaibuchi K: Purification and in vitro activity of Rho-associated kinase. Methods Enzymol 2000, 325:149-155.

34. Ishizaki T, Maekawa M, Fujisawa K, Okawa K, Iwamatsu A, Fujita A, Watanabe N, Saito Y, Kakizuka A, Morii N, Narumiya S: The small GTP-binding protein Rho binds to and activates a $160 \mathrm{kDa}$ Ser/Thr protein kinase homologous to myotonic dystrophy kinase. EMBO J 1996, 15:1885-1893.

35. Feng J, Ito M, Kureishi Y, Ichikawa K, Amano M, Isaka N, Okawa K, Iwamatsu A, Kaibuchi K, Hartshorne DJ, Nakano T: Rho-associated kinase of chicken gizzard smooth muscle. J Biol Chem 1999, 274:3744-3752.

36. Ishizaki T, Uehata M, Tamechika I, Keel J, Nonomura K, Maekawa M, Narumiya S: Pharmacological properties of Y-27632, a specific inhibitor of rho-associated kinases. Mol Pharmacol 2000, 57:976-983.

37. Sasaki Y, Suzuki M, Hidaka H: The novel and specific Rho-kinase inhibitor (S)-(+)-2-methyl-1-[(4-methyl-5-isoquinoline)sulfonyl]-homopiperazine as a probing molecule for Rho-kinase-involved pathway. Pharmacol Ther 2002, 93:225-232.

38. Wettschureck $N$, Offermanns $\mathrm{S}$ : Rho/Rho-kinase mediated signaling in physiology and pathophysiology. J Mol Med 2002, 80:629-638.

39. Ying H, Biroc SL, Li WW, Alicke B, Xuan JA, Pagila R, Ohashi Y, Okada T, Kamata $Y$, Dinter $\mathrm{H}$ : The Rho kinase inhibitor fasudil inhibits tumor 
progression in human and rat tumor models. Mol Cancer Ther 2006, 5:2158-2164.

40. Noguchi M, Hosoda K, Fujikura J, Fujimoto M, Iwakura H, Tomita T, Ishii T, Arai N, Hirata M, Ebihara K, et al: Genetic and pharmacological inhibition of Rho-associated kinase II enhances adipogenesis. J Biol Chem 2007, 282:29574-29583.

41. Liu S, Goldstein RH, Scepansky EM, Rosenblatt M: Inhibition of rho-associated kinase signaling prevents breast cancer metastasis to human bone. Cancer Res 2009, 69:8742-8751.

42. Bao W, Hu E, Tao L, Boyce R, Mirabile R, Thudium DT, Ma XL, Willette RN, Yue TL: Inhibition of Rho-kinase protects the heart against ischemia/reperfusion injury. Cardiovasc Res 2004, 61:548-558

43. Hattori T, Shimokawa H, Higashi M, Hiroki J, Mukai Y, Tsutsui H, Kaibuchi K, Takeshita A: Long-term inhibition of Rho-kinase suppresses left ventricular remodeling after myocardial infarction in mice. Circulation 2004, 109:2234-2239.

doi:10.1186/1423-0127-20-83

Cite this article as: Chuang et al: Ser1333 phosphorylation indicates ROCKI activation. Journal of Biomedical Science 2013 20:83.

\section{Submit your next manuscript to BioMed Central and take full advantage of:}

- Convenient online submission

- Thorough peer review

- No space constraints or color figure charges

- Immediate publication on acceptance

- Inclusion in PubMed, CAS, Scopus and Google Scholar

- Research which is freely available for redistribution 